\title{
A practical approach for a comprehensive evaluation and management of diabetes mellitus
}

\author{
Filippo Pieralli, ${ }^{1}$ Andrea Crociani, ${ }^{1}$ Cristina Bazzini ${ }^{2}$ \\ ${ }^{1}$ Department of Internal Medicine, University Hospital Careggi, Firenze; ${ }^{2}$ Department of Internal Medicine, Santi Cosma e \\ Damiano Hospital, Pescia (PT), Italy
}

\begin{abstract}
Diabetes mellitus (DM) is a very common disease, encountered in nearly one third of patients admitted to Internal Medicine Units. The discharge from the hospital is a crucial moment for patients with chronic and complex diseases such as DM and also for the caregiver. After hospital admission, therapeutic modifications with respect to usual therapy might have occurred, and shift to insulin therapy during admission occurs in the majority of patients. Therefore, treatment with insulin needs education of the patient and/or the caregiver for the correct drug administration and treatment of related side effects, mainly hypoglycemia. We believe that a very practical and mnemonic approach can help the clinician in the management of the delicate phase of hospital discharge of the diabetic patient.
\end{abstract}

\section{Introduction}

Diabetes mellitus (DM) is a very common disease, encountered in nearly one third of patients admitted to Internal Medicine Units. ${ }^{1}$

The discharge from the hospital is a crucial moment for patients with chronic and complex diseases such as DM and also for the caregiver. After hospital admission, therapeutic modifications with respect to usual therapy might have occurred, and shift

Correspondence: Filippo Pieralli, Department of Internal Medicine, University Hospital Careggi, Largo Brambilla 3, 50134, Firenze, Italy. Tel./Fax: +39.055.357845.

E-mail: filippopieralli@gmail.com

Key words: Diabetes; discharge.

Contributions: all authors contributed equally to the writing of the manuscript. FP had the original idea of the simplified practical approach to diabetes mellitus management and refined the final version of the manuscript; $\mathrm{AC}$ and $\mathrm{CB}$ wrote the first draft of the manuscript.

Conflict of interest: the authors declare no potential conflict of interest.

Received for publication: 8 March 2018.

Accepted for publication: 19 September 2018.

This work is licensed under a Creative Commons Attribution NonCommercial 4.0 License (CC BY-NC 4.0).

CCopyright F. Pieralli et al., 2019

Licensee PAGEPress, Italy

Italian Journal of Medicine 2019; 13:68-72

doi:10.4081/itjm.2019.1014 to insulin therapy during admission occurs in the majority of patients. Therefore, treatment with insulin needs education of the patient and/or the caregiver for the correct drug administration and treatment of related side effects, mainly hypoglycemia.

We believe that a very practical and mnemonic approach can help the clinician in the management of the delicate phase of hospital discharge of the diabetic patient (Table 1).

\section{$A$ - $A$ ssessment of cardiovascular risk and need for $A$ ntiplatelets or $A$ nticoagulant therapy}

A comprehensive evaluation of cardiovascular risk is needed, with the assessment of other comorbidities and the screening for organ damage.

Age, sex, smoking habit, blood pressure and lipid profile should be assessed to establish cardiovascular risk.

It is well known that diabetes is a very strong risk factor for cardiovascular diseases and there are many algorithms and charts for establishing cardiovascular risk. Most of them are based on populations with different basal risk profiles (e.g., based on Framingham Heart Study) or consider DM as a dichotomic variable (e.g., SCORE, Italian risk charts) without considering the burden and the duration of the disease, as well the quality of the treatment.

The United Kingdom Prospective Diabetes Study (UKPDS) outcome model may overcome the abovementioned limits (at least in type 2 patients) and it is gaining more importance as it considers both duration of the disease and the effectiveness of the treatment, furthermore it has also been validated in an Italian diabetic population. ${ }^{2,3}$

Screening for underlying asymptomatic coronary artery disease should be considered in patients with 
high pre-test probability and reasonable quality and life expectancy.

Impaired platelet function is found in diabetic patients but can be present also in conditions of insulin-resistance like prediabetes. ${ }^{4,5}$

Aspirin (75-160 mg/day) is indicated in patients with a previous cardiovascular event while clopidogrel ( $75 \mathrm{mg} /$ day) can be used in patients intolerant to aspirin.

Clopidogrel is preferred to aspirin in patients with clinical peripheral artery disease. ${ }^{6,7}$

To date, there is a lack of evidence about the net benefit of treating patients suffering from DM with antiplatelet agents in primary prevention.

Recent guidelines ${ }^{3,8}$ suggest that it could be considered in high risk patients (age $>50$ years and with at least one additional major risk factor among family history of premature atherosclerotic cardiovascular disease, hypertension, dyslipidemia, smoking, or albuminuria) without high bleeding risk.

Diabetes mellitus, counting for 1 point, is included in the $\mathrm{CHA}_{2} \mathrm{DS}_{2}$-VASc score for the stratification of the risk of cerebrovascular events in patients with atrial fibrillation.

\section{B - Blood pressure control targets and medications}

Since hypertension is often found in diabetic patients, and it is usually as a sign of underlying nephropathy in type 1 diabetes and as a comorbidity in type 2 (up to $80 \%$ ), blood pressure should be measured at every check-up.

Clinical trials ${ }^{9,10}$ and meta-analysis ${ }^{11}$ have shown that lowering blood pressure is associated with a significant reduction in overall mortality and cardiovascular events in diabetic patients.

The antagonism of the renin-angiotensin system is the cornerstone of the treatment of hypertension in diabetic patients as it has shown also its efficacy in the prevention of the nephropathy. ${ }^{12}$ Therefore an angiotensin converting enzyme inhibitor or, if not tolerated, an angiotensin receptor blocker, in addition to lifestyle changes, is the first-line treatment especially in case of proteinuria or microalbuminuria. ${ }^{13,14}$

Simultaneous administration of two blockers of the renin angiotensin system (RAS) is not recommended and should be avoided in patients with diabetes due to the risk of significant hypotension and hyperkaliemia.

Thiazide diuretics and calcium antagonists have been shown to be useful, especially when combined with a RAS blocker.

$\beta$-blockers, though potentially impairing insulin sensitivity, are useful for BP control in combination therapy, especially in patients with coronary heart disease and heart failure. ${ }^{13}$

Most patients should be treated to a systolic goal of $<140 \mathrm{mmHg}$ and a diastolic goal of $<90 \mathrm{mmHg}$.

Although with a weak recommendation, lower targets $(<130 / 80 \mathrm{mmHg})$ might be considered in highrisk patients.

Less intensive control $(<150 / 90 \mathrm{mmHg})$ should be considered in older patients, those at high risk of falls, and poor life expectancy.

\section{C - Cholesterol treatment targets and lipid lowering agents}

Fasting lipid profile should be assessed yearly as diabetic patients are often subject to lipid profile alterations, which contribute to the increase of cardiovascular risk.

The reduction of the low-density lipoprotein (LDL) shows benefits in term of both global (9\%) and cardiovascular (21\%) mortality. ${ }^{15}$

Lifestyle modification (high-fiber and low-fat diet, physical activity) should be recommended to improve the lipid profile.

Statin therapy remains the first-line treatment in patients with type-1 diabetes or in type 2 above the age of 40 years $^{14}$ with LDL out of target despite lifestyle intervention, as they are well tolerated and, with the exception of muscular symptoms, adverse effects are rare.

LDL reduction is the main goal with the following considerations: i) LDL reduction in type-1 diabetic patients with microalbuminuria and/or renal disease should be at least $50 \%$ irrespective of the baseline LDL concentration; ii) for most type-2 diabetic patients LDL reduction target should be $<100 \mathrm{mg} / \mathrm{dL}$ while a lower goal of $<70 \mathrm{mg} / \mathrm{dL}$ should be considered

Table 1. Phases of hospital discharge of the diabetic patient.

\begin{tabular}{ll}
\hline$A$ & Assessment of cardiovascular risk and need for $A$ ntiplatelets or $A$ nticoagulant therapy \\
\hline$B$ & Blood pressure control targets and medications \\
\hline$C$ & Cholesterol treatment targets and lipid lowering agents \\
\hline$D$ & Diabetes control evaluation, Diabetic, Drugs choice \\
\hline$E$ & Education: lifestyle, training, diet, tobacco cessation, Exercise \\
\hline$F$ & Follow-up
\end{tabular}


in very high-risk patients (SCORE calculated $\geq 10 \%$ or previous cardiovascular events).

Further targets can be considered for high-density lipoprotein (HDL) cholesterol ( $>40 \mathrm{mg} / \mathrm{dL}$ for men; $>50$ for women $\mathrm{mg} / \mathrm{dL}$ ) and triglyceride levels (TGL $<150 \mathrm{mg} / \mathrm{dL}$ ).

Addition of ezetimibe should be considered in case of statin therapy failure or intolerance ${ }^{3}$ or in case of recent acute coronary syndrome and LDL $>50$ $\mathrm{mg} / \mathrm{dL} .{ }^{16}$

The combination statin/fibrate is generally not recommended but it might be considered for men with both TGL $>204$ and HDL $<34 \mathrm{mg} / \mathrm{dL}$. Proprotein convertase subtilisin/kexin type 9 inhibitors will be an alternative or combined option for lowering cholesterol levels in high-risk patients in the next future. ${ }^{3}$

\section{$D$ - Diabetes control evaluation and anti-diabetic Drugs choice}

Short-term control should be evaluated by monitoring glucose serum levels with different targets based on different setting.

In-hospital critically ill patients should maintain levels between 140 and $180 \mathrm{mg} / \mathrm{dL}$ while other patients' targets for fasting, preprandial and postprandial glucose should be $90-130 \mathrm{mg} / \mathrm{dL},<140$ $\mathrm{mg} / \mathrm{dL}$ and $<180 \mathrm{mg} / \mathrm{dL}$ respectively. ${ }^{17}$

Medium-term control is evaluated by $\mathrm{HbA} 1 \mathrm{c}$ monitoring. A reasonable goal for adults is $7 \%(53$ $\mathrm{mmol} / \mathrm{mol}$ ) while for selected patients (e.g., short duration of disease, type 2 diabetes treated with lifestyle or metformin only, long life expectancy, no significant cardiovascular disease) a lower goal $(6.5 \%, 48$ $\mathrm{mmol} / \mathrm{mol}$ ), wherever possible, should be considered.

By the way, patients with a history of severe hypoglycemia, poor life expectancy, advanced vascular complications, relevant comorbidities or long course of the disease should be considered for a less stringent goal of $\mathrm{HbA} 1 \mathrm{c}(8 \%$ or $64 \mathrm{mmol} / \mathrm{mol})$ in order to avoid hypoglycemic episodes which can have detrimental effects in such fragile population. ${ }^{8}$

At discharge, glycemic targets are $90-130 \mathrm{mg} / \mathrm{dL}$ for fasting glucose and 90-150 for bedtime glucose; less stringent targets (90-150 for fasting glucose and 100$180 \mathrm{mg} / \mathrm{dL}$ for bedtime glucose or 100-180 and 110-200 $\mathrm{mg} / \mathrm{dL}$ ) for complex patients with multiple chronic illnesses and with moderate to severe cognitive impairment or major functional disability., 3

Metformin, if tolerated, is recommended as the first-line therapy in type 2 diabetic patients.

In recent years the landscape of pharmacological treatment of type $2 \mathrm{DM}$ has dramatically enriched. Nowadays, many drugs are available giving the possibility to a tailored therapy.

Some drugs have shown a significant favorable impact on cardiovascular outcomes and in some cases on overall mortality. Among these, pioglitazone, which is contraindicated in heart failure, has been shown to reduce the composite end-point of all-cause mortality, non-fatal myocardial infarction, and stroke in patients with type 2 diabetes who are at high risk of macrovascular events. ${ }^{18}$

Many beneficial effects of incretins have been described with a favorable pharmacological profile in terms of efficacy and safety.

Many trials are ongoing and some trials and metaanalysis have shown at least a neutral effect of DPP-4 inhibitors on cardiovascular outcome, while GLP-1 agonists, notably have been shown to be able to reduce the cardiovascular mortality and major cardiovascular events in patients with type 2 diabetes and high cardiovascular risk. ${ }^{19}$

SGLT-2 inhibitors are promising molecules for reducing the risk of cardiovascular events in patients with type $2 \mathrm{DM}$ and elevated cardiovascular risk. In particular, empagliflozin has been shown to reduce cardiovascular and total mortality and should be considered early in the course of the disease. ${ }^{20}$

In a recently published trial involving patients with type 2 diabetes and an elevated risk of cardiovascular disease, patients treated with canagliflozin had a lower risk of cardiovascular events than those who received placebo, but a greater risk of foot amputation. ${ }^{21}$

Despite drugs advancements, to date insulin therapy remains the gold standard for in-hospital management for many reasons: i) the high probability, especially in critically ill patients, of not being able to take oral medication; ii) the need to stop oral agents, in particular metformin, for the execution of instrumental investigations with administration of contrast medium; iii) the presence of acute comorbidities such as renal, hepatic or cardiac failure which may contraindicate the use of some non-insulin drugs (e.g., metformin and thiazolidinedione); iv) the extreme flexibility of insulin therapy that makes it adaptable to the dynamic clinical situation of the hospitalized patient (changes in meal times, reduction of food intake, fasting for instrumental investigations or surgery); v) the possibility of adjusting the dose of insulin therapy individually on the basis of clinical history, co-morbidities present upon hospital admission, weight and the risk of hypoglycemia. ${ }^{3}$

At discharge the type of medication (e.g., insulin $v s$ other agents or association therapy) could be chosen on the basis of HbAlc levels, with a view on treatment preceding hospitalization, presence of new comorbidities, glucose control during in hospital stay, other medications (e.g., corticosteroid therapy) and type of discharge (e.g., home, rehabilitation or nursing facilities).

As an example, a HbAlc based protocol has been 
proposed $^{22}$ as follows: i) patients with $\mathrm{HbAlc}$ at admission $<7 \%$ can be discharged with the same preadmission therapy, if not otherwise contraindicated; ii) patients with $\mathrm{HbAlc}$ between 7 and $9 \%$ can be discharged with oral agents plus half of inpatient basal insulin dose; iii) patients with $\mathrm{HbAlc}>9 \%$ can be discharged with oral agents plus $80 \%$ of inpatient basal insulin dose or with a basal bolus insulin regimen.

\section{$E$ - Education: lifestyle, training, diet, tobacco cessation, Exercise}

While an exhaustive education is not feasible during the hospital stay, a basic knowledge should be shared with the patient and/or caregivers before the discharge.

Healthcare professionals should train and educate the patient and the caregiver with some basics considering age, cognitive abilities, socio-cultural and economic situation, and daily activities.

A seven-skill scheme is provided by the American Association of Diabetes Educators as a model of simple and practical tool to deal with diabetes. ${ }^{23}$

The following seven items should be shared with patients and caregivers in a simple and explicative manner.

Healthy eating: Make sure the food contains the right amounts of carbohydrates, protein and fat, eat on schedule and watch the salt intake.

Being active: It has multiple health benefits like losing weight, lowering cholesterol, improving blood pressure and lowering stress and anxiety hence improving the mood.

Monitoring: Checking blood glucose levels is fundamental to reach treatment goals and to make food, therapy and activity modifications.

Taking medications: There are several types of medications that are often prescribed for people with diabetes like insulin, oral agents, aspirin, blood pressure medication, cholesterol-lowering medication, etc., in order to reduce the pathologic burden. Patient and/or caregiver should be aware of their role and major side effects in order to maximize therapeutic compliance.

Problem solving: Basics about short-term complications (e.g., hypoglycemia) management should be given. A simple protocol for dealing with hypoglycemia is represented by the rule of 15 in which 15 grams of sugar (i.e., equal to 3 sugar teaspoons, to half coke can or a glass of fruit juice) are given followed by blood glucose control after $15 \mathrm{~min}$ and repeated until reaching a level of glucose above $100 \mathrm{mg} / \mathrm{dL} .{ }^{17}$

Risk reduction: Information about diabetes-related complications should be given and cholesterol, blood pressure, feet, teeth and eyes should be examined regularly.

Healthy coping: Dealing with diabetes can be tiresome and frustrating. It is important to learn ways to stay motivated and to recognize negative emotions to reduce their negative impact.

\section{F - Follow-up}

At discharge, in general, patients with newly diagnosed diabetes or with non-optimal diabetes control (HbAlc greater than $8 \%$ ) or severe diabetic complications should be referred within one month to an outpatient diabetes care center for a new evaluation and follow-up. ${ }^{3,8}$ This is a general rule that should be adapted to the single patient according to age, cognitive and functional status, comorbidities and life expectancy.

Reassessment of the comorbidities and the screening for organ damage through medical history, physical examination, laboratory and instrumental evaluation should be repeated on a scheduled basis: i) accurate medical history focusing on cardiovascular family history, cardiovascular risk factors and events and signs or symptoms related to acute and chronic complications; ii) blood pressure (at both arms), heart rate, waist circumference and body mass index; iii) physical examination (in particular cardiovascular system, legs and feet examination); iv) HbAlc (if not available in the past 3 months; then at least two times a year in patients who are meeting treatment goals and who have stable glycemic control; quarterly in patients whose therapy has changed or who are not meeting glycemic goals); v) evaluation of renal and liver function, albuminuria and fasting lipid profile (at least yearly); vi) screening for neuropathy (both sensory and autonomic including sexual disfunction and gastroparesis) if symptoms are present or on the basis of diabetic neuropathy index with clinical examination; vii) electrocardiogram (yearly) and echocardiography (every 3 years if normal); viii) carotid duplex and ankle brachial index (every 3 years if normal); ix) fundoscopic examination (at least yearly).

\section{References}

1. Pieralli F, Bazzini C, Fabbri A, et al. The classification of hospitalized patients with hyperglycemia and its implication on outcome: results from a prospective observational study in Internal Medicine. Intern Emerg Medicine 2016;11:649-56.

2. Pagano E, Gray A, Rosato R, et al. Prediction of mortality and macrovascular complications in type 2 diabetes: validation of the UKPDS outcomes model in the Casale Monferrato Survey, Italy. Diabetologia 2013;56:1726-34.

3. Associazione Medici Diabetologi (AMD) - Società Italiana di Diabetologia (SID). Standard italiani per la cura del diabete mellito; 2016. Available from: http://www.standarditaliani.it

4. Davi G, Catalano I, Averna M, et al. Thromboxane biosynthesis and platelet function in type II diabetes mellitus. N Engl J Med 1990;322:1769-74. 
5. Santilli F, Formoso G, Sbraccia P, et al. Postprandial hyperglycemia is a determinant of platelet activation in early type 2 diabetes mellitus. J Thromb Haemost 2010;8:828-37.

6. Creager MA. Results of the CAPRIE trial: efficacy and safety of clopidogrel. Clopidogrel versus aspirin in patients at risk of ischaemic events. Vasc Med 1998;3:257-60.

7. Aboyans V, Ricco JB, Bartelink ML, et al. 2017 ESC Guidelines on the Diagnosis and Treatment of Peripheral Arterial Diseases, in collaboration with the European Society for Vascular Surgery (ESVS). Eur Heart J 2017;00:1-60.

8. American Diabetes Association. Standards of medical care in diabetes - January 2017. Diabetes Care 2017;40: Supplement 1. Available from: http://care.diabetes journals.org/content/diacare/suppl/2016/12/15/40.Supple ment_1.DC1/DC_40_S1_final.pdf

9. Patel A, MacMahon S, Chalmers J, et al. Effects of a fixed combination of perindopril and indapamide on macrovascular and microvascular outcomes in patients with type 2 diabetes mellitus (the ADVANCE trial): a randomised controlled trial. Lancet 2007;370:829-40.

10. UK Prospective Diabetes Study Group. Tight blood pressure control and risk of macrovascular and microvascular complications in type 2 diabetes: UKPDS 38. BMJ 1998;317:703.

11. Reboldi G, Gentile G, Angeli F, et al. Effects of intensive blood pressure reduction on myocardial infarction and stroke in diabetes: a meta-analysis in 73,913 patients. J Hypertens 2011;29:1253-69.

12. Ruggenenti P, Fassi A, Ilieva AP, et al. Bergamo Nephrologic diabetes. Complications Trial (BENEDICT) Investigators. Preventing microalbuminuria in type 2 diabetes. N Engl J Med 2004;351:1941-51.

13. Mancia G, Fagard R, Narkiewicz K, et al. 2013 ESH/ESC Guidelines for the management of arterial hypertension. Eur Heart J 2013;34:2159-219.

14. Piepoli MF, Hoes AW, Agewall S, et al. 2016 European
Guidelines on cardiovascular disease prevention in clinical practice. Eur Heart J 2016;37:2315-81.

15. Kearney PM, Blackwell L, Collins R, et al. for Cholesterol Treatment Trialists' (CTT) Collaborators. Efficacy of cholesterol-lowering therapy in 18686 people with diabetes in 14 randomized trials of statins: a meta-analysis. Lancet 2008;371:117-25.

16. Cannon CP, Blazing MA, Giugliano RP, et al.; for the IMPROVE-IT Investigators. Ezetimibe added to statin therapy after acute coronary syndromes. N Engl J Med 2015;372:2387-97.

17. Beltramello G, Manicardi V, Trevisan R. Trialogue. Managing hyperglycemia in internal medicine: instructions for use. Acta Diabetol 2013;50:465-73.

18. Dormandy JA, Charbonnel B, Eckland DJ, et al. Secondary prevention of macrovascular events in patients with type 2 diabetes in the PROactive Study (PRO spectivepioglitAzone Clinical Trial In macroVascular Events): a randomised controlled trial. Lancet 2005;366:1279-89.

19. Marso SP, Daniels GH, Brown-Frandsen K, et al.; LEADER Trial Investigators. Liraglutide and cardiovascular outcomes in type 2 diabetes. N Engl J Med 2016;375:311-22.

20. Zinman B, Wanner C, Lachin JM, et al.; EMPA-REG OUTCOME Investigators. Empagliflozin, cardiovascular outcomes, and mortality in type 2 diabetes. N Engl J Med 2015;373:2117-28.

21. Neal B, Perkovic V, Mahaffey KW, et al. Canagliflozin and cardiovascular and renal events in type 2 diabetes. N Engl J Med 2017;377:644-57.

22. Umpierrez GE, Reyes D, Smiley D, et al. Hospital discharge algorithm based on admission $\mathrm{HbA}_{1 \mathrm{c}}$ for the management of patients with type 2 diabetes. Diabetes Care 2014;37:2934-9.

23. American Association of Diabetes Educators. AADE7 self-care behaviors ${ }^{\circledR}$. Available from: https://www. diabeteseducator.org/living-with-diabetes/aade7-self-care -behaviors 